SOUTH DAKOTA

// The Geology of Shale Gas and Tight Oil Resources in the United States Daniel J. Soeder

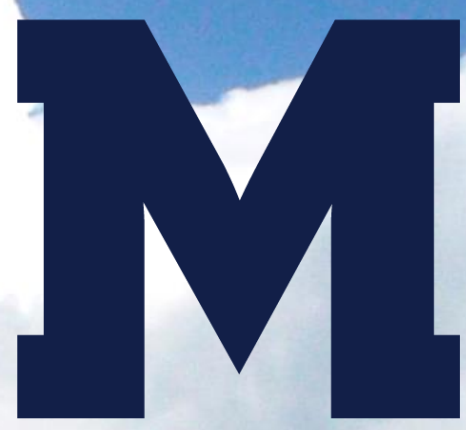

SCHOOL OF MINES $\&$ TECHNOLOGY

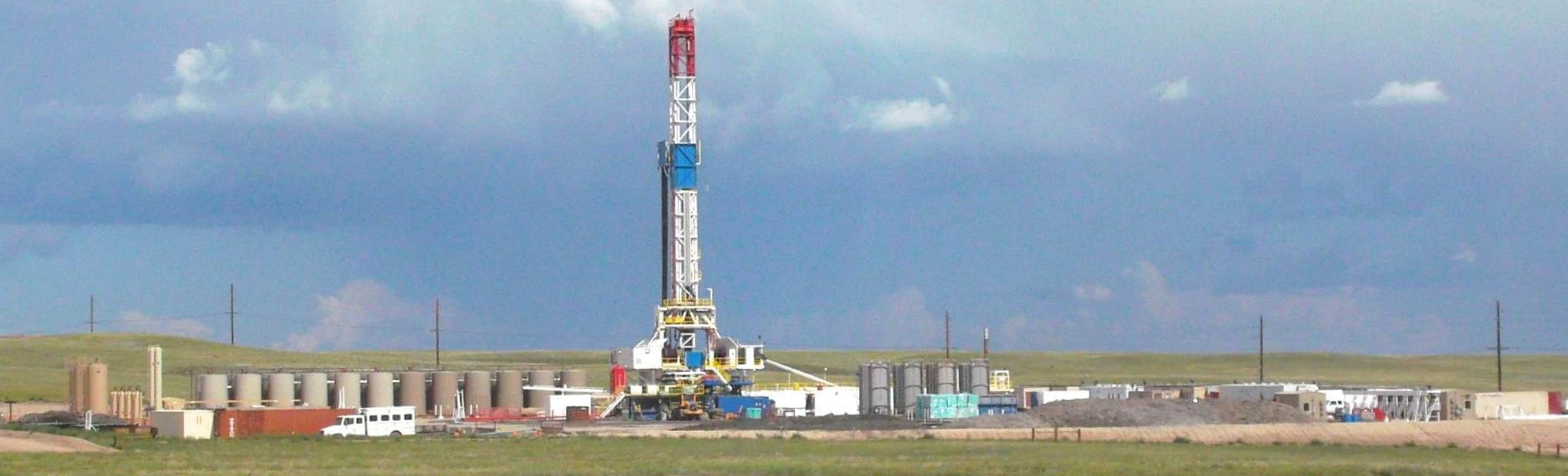


GSA Annual Meeting, November 4-7, 2018, Indianapolis, Indiana

T34. Unconventional Energy Resources (GSA Energy Geology Division) Abstract ID: 320783

\section{THE GEOLOGY OF SHALE GAS AND TIGHT OIL RESOURCES IN THE UNITED STATES}

Daniel J. Soeder, South Dakota School of Mines \& Technology, Rapid City, SD

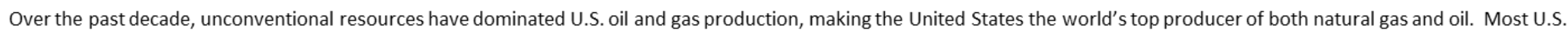

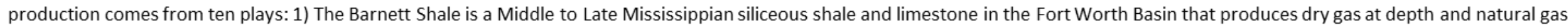

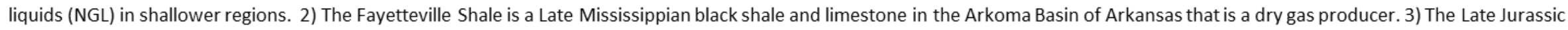

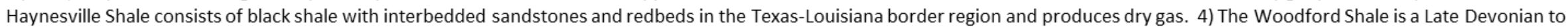

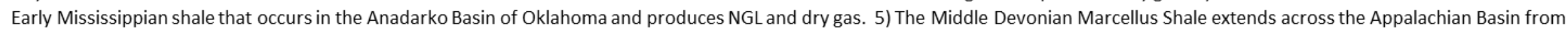

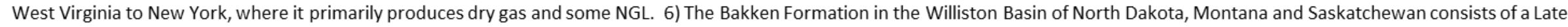

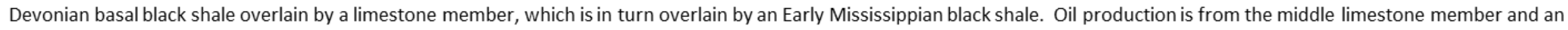

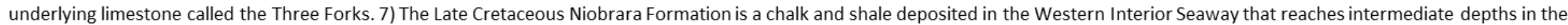

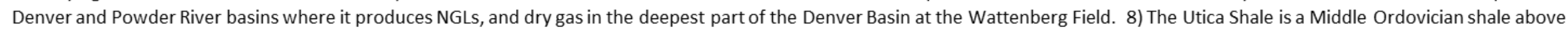

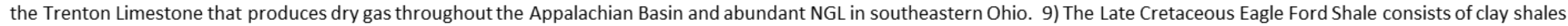

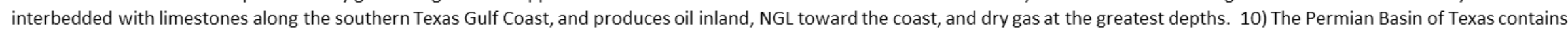

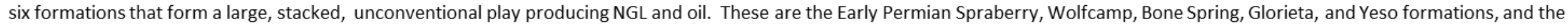
Middle Permian Delaware Mountain Group. Understanding the geologic factors that affect the behavior of shales can help to improve predictability of resource recovery.

(Dan.Soeder@sdsmt.edu) 


\section{// Shale Gas Origins}

In the United States

Fall 1973 to Spring 1974: "Energy Crisis"

- October: Yom Kippur war - U.S. supported Israel

- OPEC Arab states embargoed oil exports to U.S.

- Price of gasoline quadrupled $(\$ 0.40-\$ 1.60)$

- Gasoline was in short supply, nearly rationed

U.S. Department of Energy established in 1977 to fund domestic energy R\&D projects.

- Eastern gas shales project (EGSP) 1976-92

- Western tight gas sands

- Coalbed methane

- Geopressured aquifers/ultra-deep gas

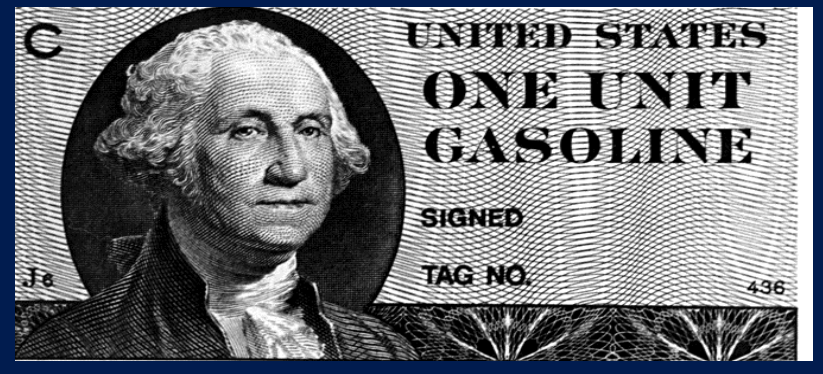

Photos: public archives

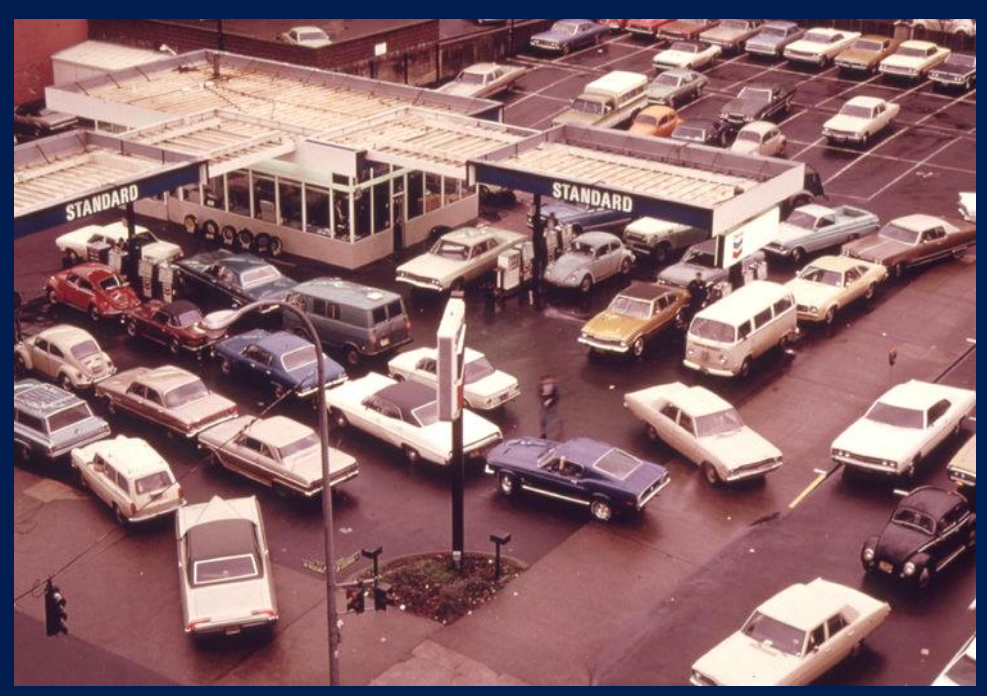

EGSP focus: 1) define the resource, 2) develop the engineering, 3) transfer the technology

A second energy crisis in 1979 following the Iranian revolution further heightened the need. 


\section{// Shale Gas Origins}

In the United States

U.S. shale gas has a history of small-scale production going back to the $19^{\text {th }}$ Century.

Engineering/economic challenges of EGSP

- Oversimplified concept of black shales + fractures $=$ gas. Some shales produced gas and others did not.

- EGSP conceptual model was the Big Sandy Field in Kentucky, which is unique.

- Stimulation treatments were hit or miss for unknown reasons.

Mitchell Energy continued experimenting with drilling and completion techniques on the Barnett Shale in Texas post-EGSP, driving shale revolution.

- Mitchell success on the Barnett in 1997.

- Southwestern success on Fayetteville in 2004.

- Chesapeake developed the Haynesville in 2005.

- Range Resources success on Marcellus 2007.

Schrider, L. A. and Wise, R.L., 1980, Potential new sources of natural gas: Journal of Petroleum Technology, April 1980, p. 703-716. Photos at right by Dan Soeder.

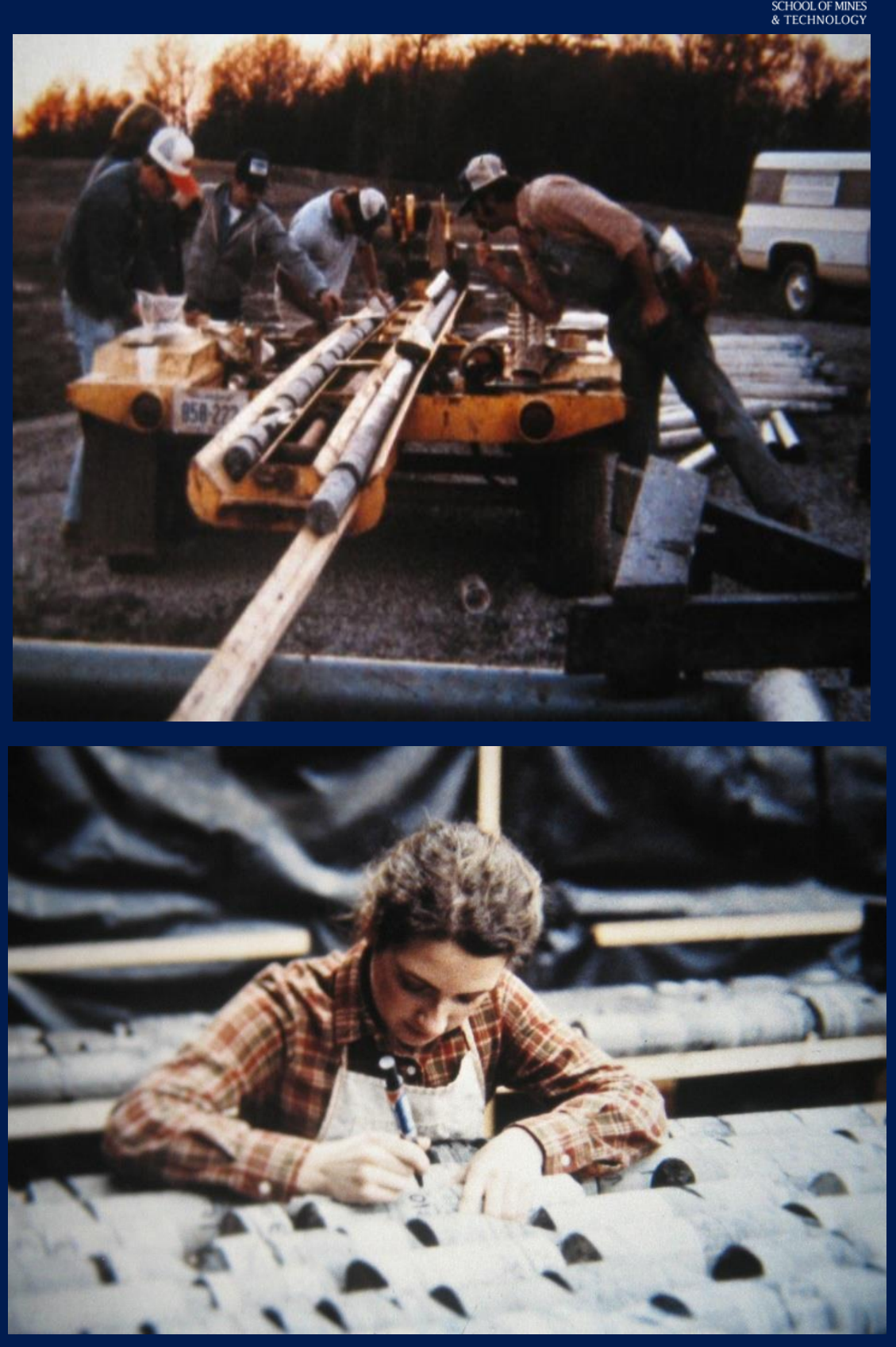




\section{// Conventional vs. Unconventional}

The Geology of Conventional and Unconventional Oil and Gas

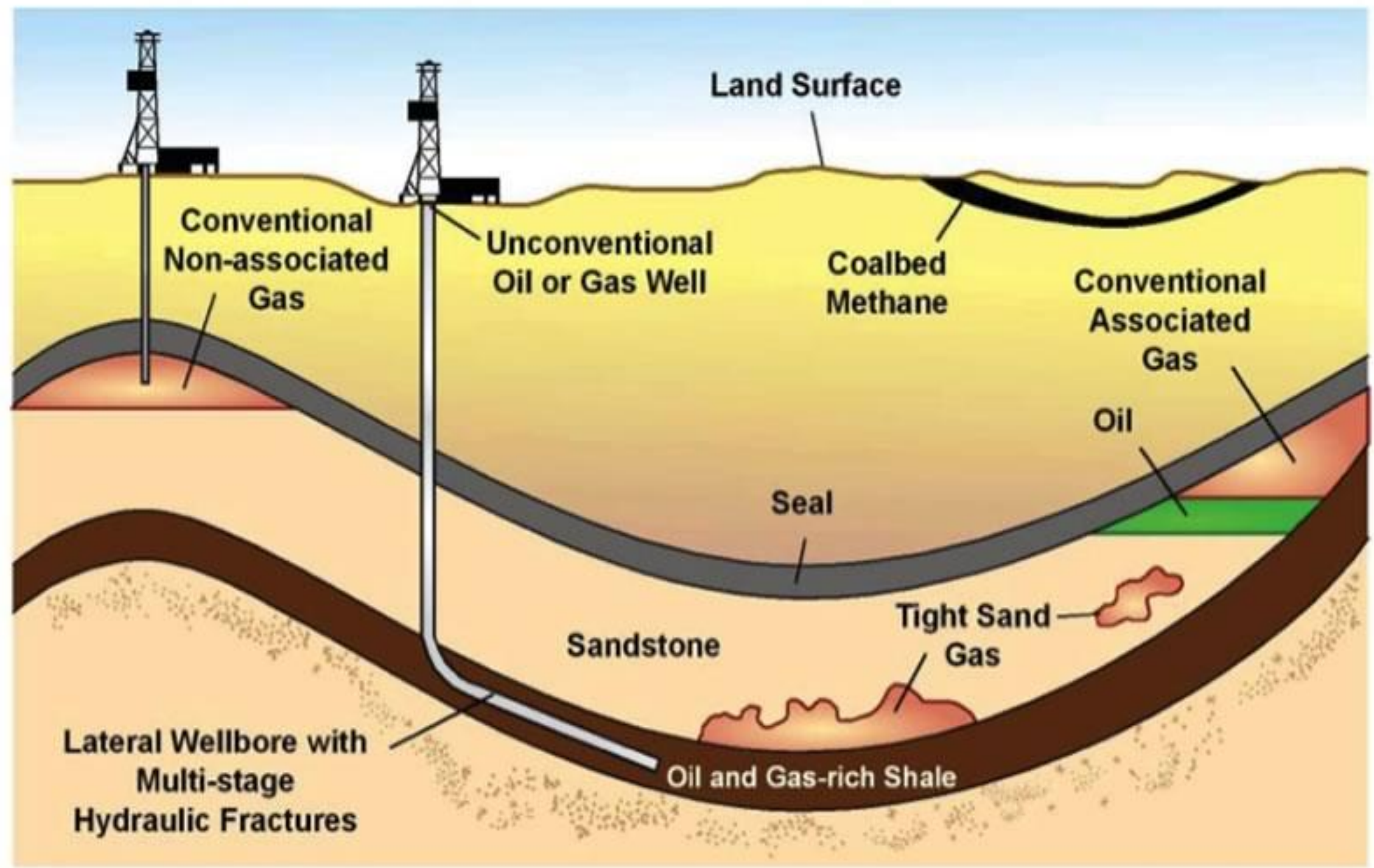




\section{// Shale Gas Development}

\section{Ten Major Plays}

\begin{tabular}{|c|c|c|c|c|c|c|c|}
\hline \multirow[b]{2}{*}{ Formation } & \multirow[b]{2}{*}{ Age } & \multicolumn{4}{|c|}{ Summary of U.S. Shale Plays } & \multirow[b]{2}{*}{ Production } & \multirow[b]{2}{*}{ Core Areas } \\
\hline & & Basins \& Location & Primary Developer & Year & Depth & & \\
\hline Barnett Shale & Mid to Late Miss & Fort Worth, TX & Mitchell Energy & 1997 & $0-8 \mathrm{kft}$ & gas, NGL & $\begin{array}{l}\text { Newark East } \\
\text { Field; NW of Ft. } \\
\text { Worth }\end{array}$ \\
\hline Fayetteville Shale & Late Miss & Arkoma, AR & Southwest Energy & 2004 & $0-6 \mathrm{ktt}$ & dry gas & $\begin{array}{l}\text { North-central } \\
\text { Arkansas }\end{array}$ \\
\hline Haynesville-Bossier & Late Jurassic & Arkla, TX-LA & $\begin{array}{c}\text { Chesapeake } \\
\text { Energy }\end{array}$ & 2005 & $10 \mathrm{k}-13 \mathrm{kft}$ & dry gas & $\begin{array}{l}\text { Lufkin, TX to } \\
\text { Shreveport, LA }\end{array}$ \\
\hline Marcellus Shale & Mid Devonian & $\begin{array}{c}\text { Appalachian, WV, } \\
\text { PA }\end{array}$ & Range Resources & 2007 & $0-9 \mathrm{ktt}$ & gas, NGL & $\begin{array}{l}\text { SW PA \& NW WV; } \\
\text { NE PA }\end{array}$ \\
\hline Bakken Formation & $\begin{array}{c}\text { Late Devonian to } \\
\text { Early Miss }\end{array}$ & $\begin{array}{c}\text { Williston, ND, MT, } \\
\text { SK }\end{array}$ & EOG Resources & $\begin{array}{c}2006- \\
2009\end{array}$ & $4 \mathrm{k}-11 \mathrm{kft}$ & oil, gas & $\begin{array}{l}\text { NW North } \\
\text { Dakota, E. } \\
\text { Montana, } \\
\text { Canada. }\end{array}$ \\
\hline Woodford Shale & Late Devonian & $\begin{array}{c}\text { Anadarko, } \\
\text { Ardmore, OK }\end{array}$ & $\begin{array}{l}\text { Newfield } \\
\text { Exploration }\end{array}$ & 2005 & $4 k-25 k f t$ & oil, NGL, dry gas & $\begin{array}{l}\text { central \& } \\
\text { southern } \\
\text { Oklahoma }\end{array}$ \\
\hline Niobrara Formation & Late Cretaceous & $\begin{array}{l}\text { Denver; Powder } \\
\text { River, CO, WY }\end{array}$ & $\begin{array}{l}\text { Whiting } \\
\text { Petroleum }\end{array}$ & 2008 & $0-11 \mathrm{kft}$ & NGL, dry gas & $\begin{array}{l}\text { E. Colorado, E. } \\
\text { Wyoming }\end{array}$ \\
\hline Eagle Ford Shale & Late Cretaceous & TX Gulf Coast & Petrohawk Energy & 2008 & $0-20 \mathrm{ktt}$ & oil, NGL, gas & southern Texas \\
\hline $\begin{array}{l}\text { Spraberry, } \\
\text { Wolfcamp, Bone } \\
\text { Spring, Glorieta, } \\
\text { Yeso, and Delaware } \\
\text { formations. }\end{array}$ & Mid to Late Permian & Permian, TX-NM & Multiple & 2009 & $\sim 1 \mathrm{k}-25 \mathrm{kft}$ & oil, NGL, gas & $\begin{array}{l}\text { West Texas, SE } \\
\text { New Mexico }\end{array}$ \\
\hline Utica/Point Pleasant & Mid Ordovician & Appalachian, $\mathrm{OH}$ & Multiple & 2011 & $0-15 k \mathrm{ft}$ & gas, NGL & southeast Ohio \\
\hline
\end{tabular}




\section{// Shale Resources - North America}

2011

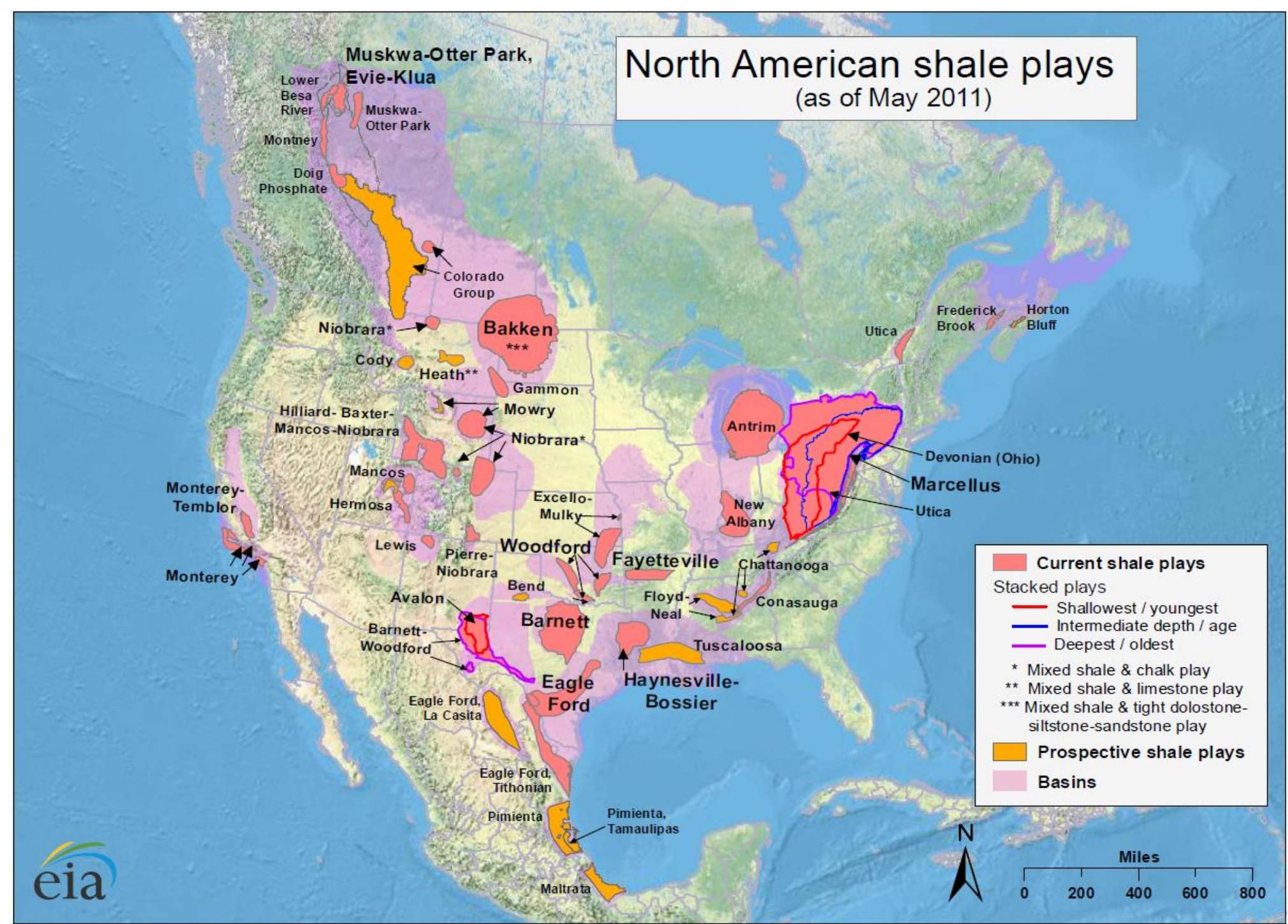

Source: U.S. Energy Information Administration based on data from various published studies. Canada and Mexico plays from ARI Updated: May 9, 2011 


\section{// 1. Barnett Shale} Fort Worth Basin

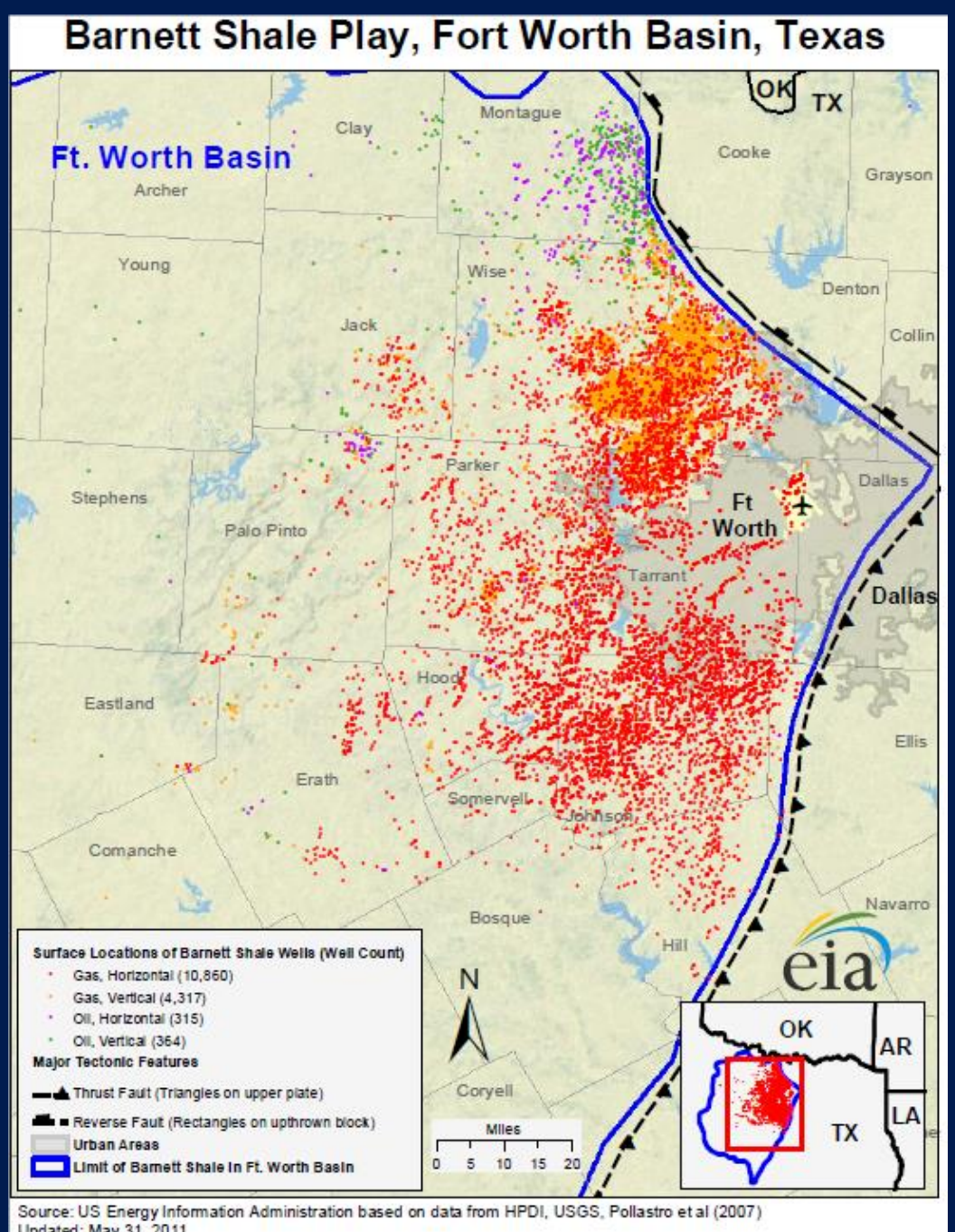

- Middle to Late Mississippian siliceous black shale and limestone.

- In 1997 Mitchell Energy successfully applied horizontal drilling $(5,000 \mathrm{ft})$ and staged hydraulic fracturing (10).

- Basin is deeper to the northeast and shallower south on the Llano uplift.

- Shale produces dry gas at depth and NGL in shallower regions.

Photo from Bruner \& Smosna, 2011

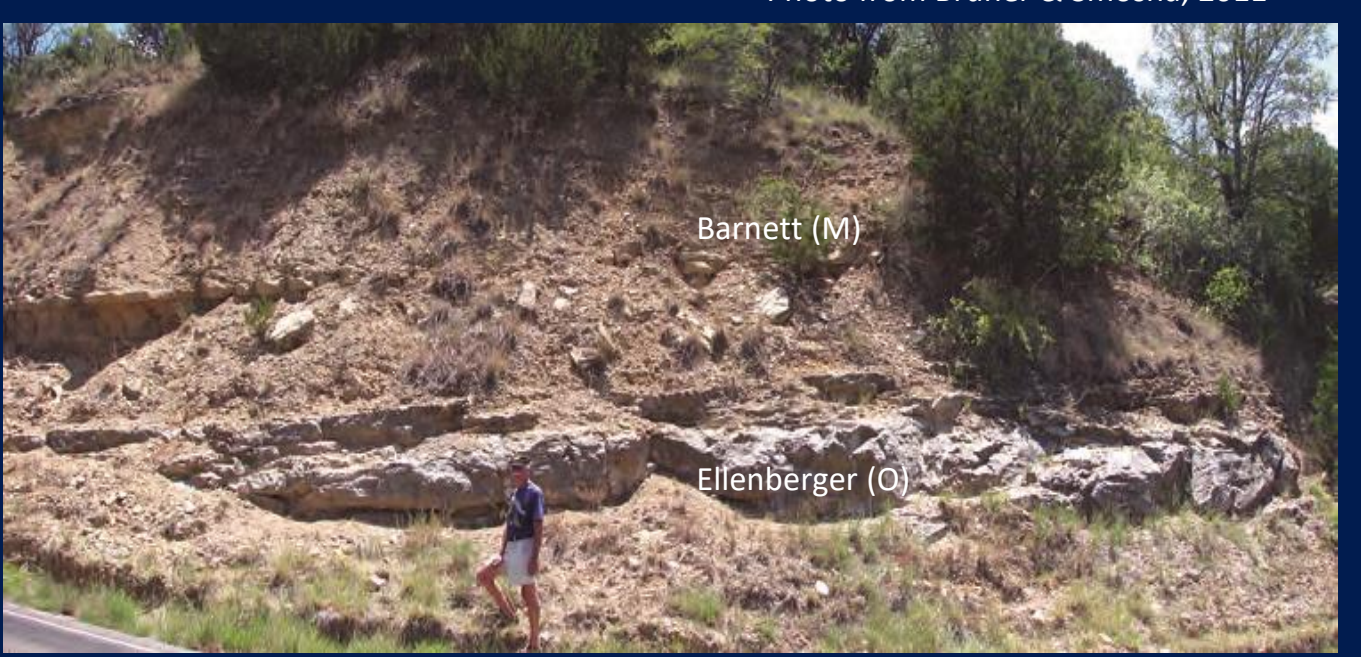




\section{// 2. Fayetteville Shale}

\section{Arkoma Basin}

- Late Mississippian fissile black shale and interbedded dark limestone, similar to Barnett.

- Developed in 2004 by Southwestern Energy using Mitchell's techniques from the Barnett.

- Main production in north central Arkansas.

- Thermally mature; dry gas producer.
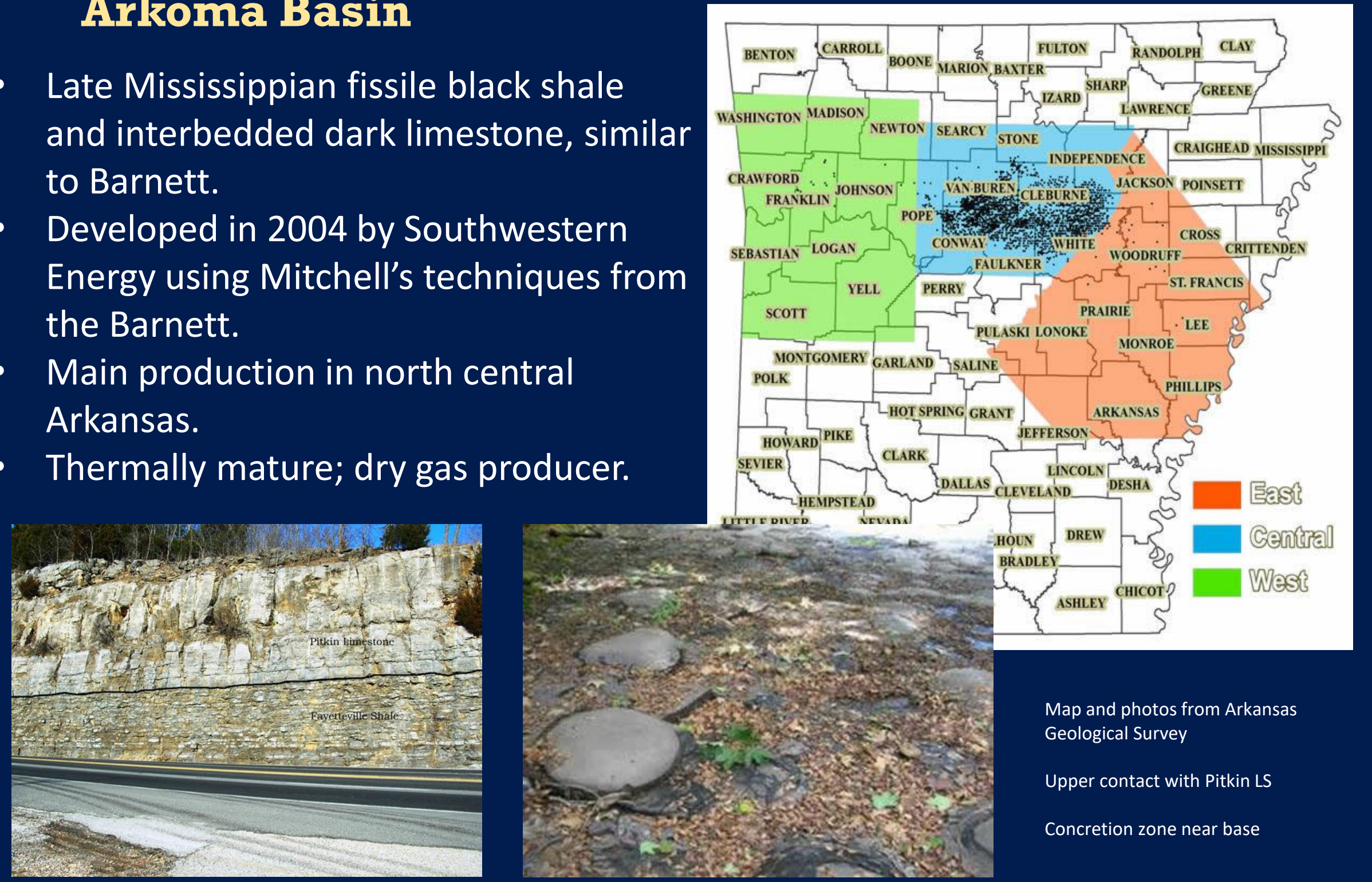

Map and photos from Arkansas Geological Survey

Upper contact with Pitkin LS 


\section{// 3. Haynesville-Bossier Shale}

\section{Arkla Basin}

- Late Jurassic marine black shale with interbedded sandstones and redbeds.

- Located 3-4 km subsurface (no outcrop) in the Texas-Louisiana border region.

- Thermally mature, produces dry gas; developed by Chesapeake in 2004.

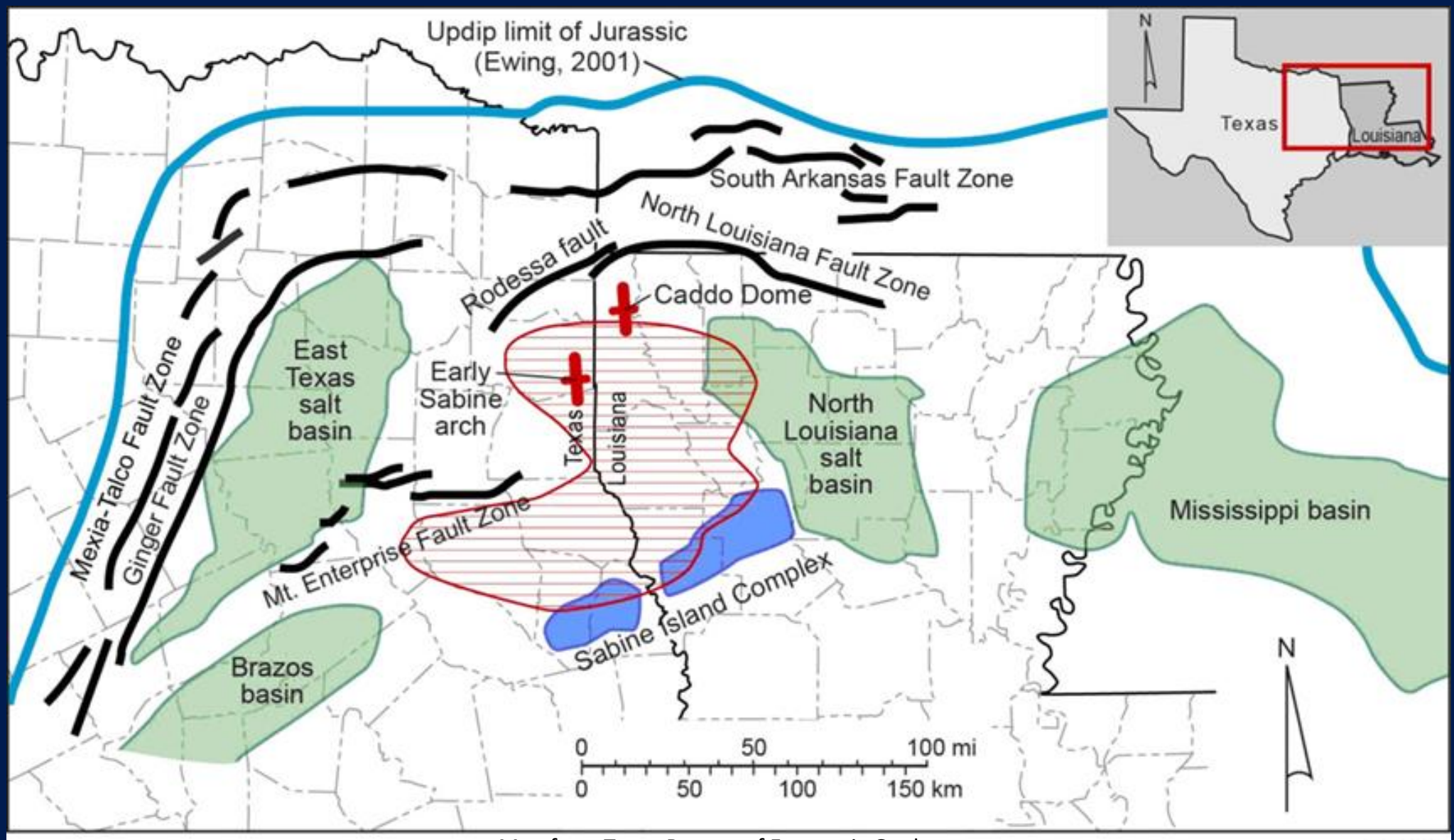




\section{// 4. Marcellus Shale}

Appalachian Basin

- Middle Devonian black, siliceous shale, middle carbonate, upper clay shale.

- Depth varies across basin; production primarily dry gas; some NGL near Ohio.

- Range Resources was the primary developer.

- Late Devonian shales were main target of EGSP; only a few cores reached Marcellus.

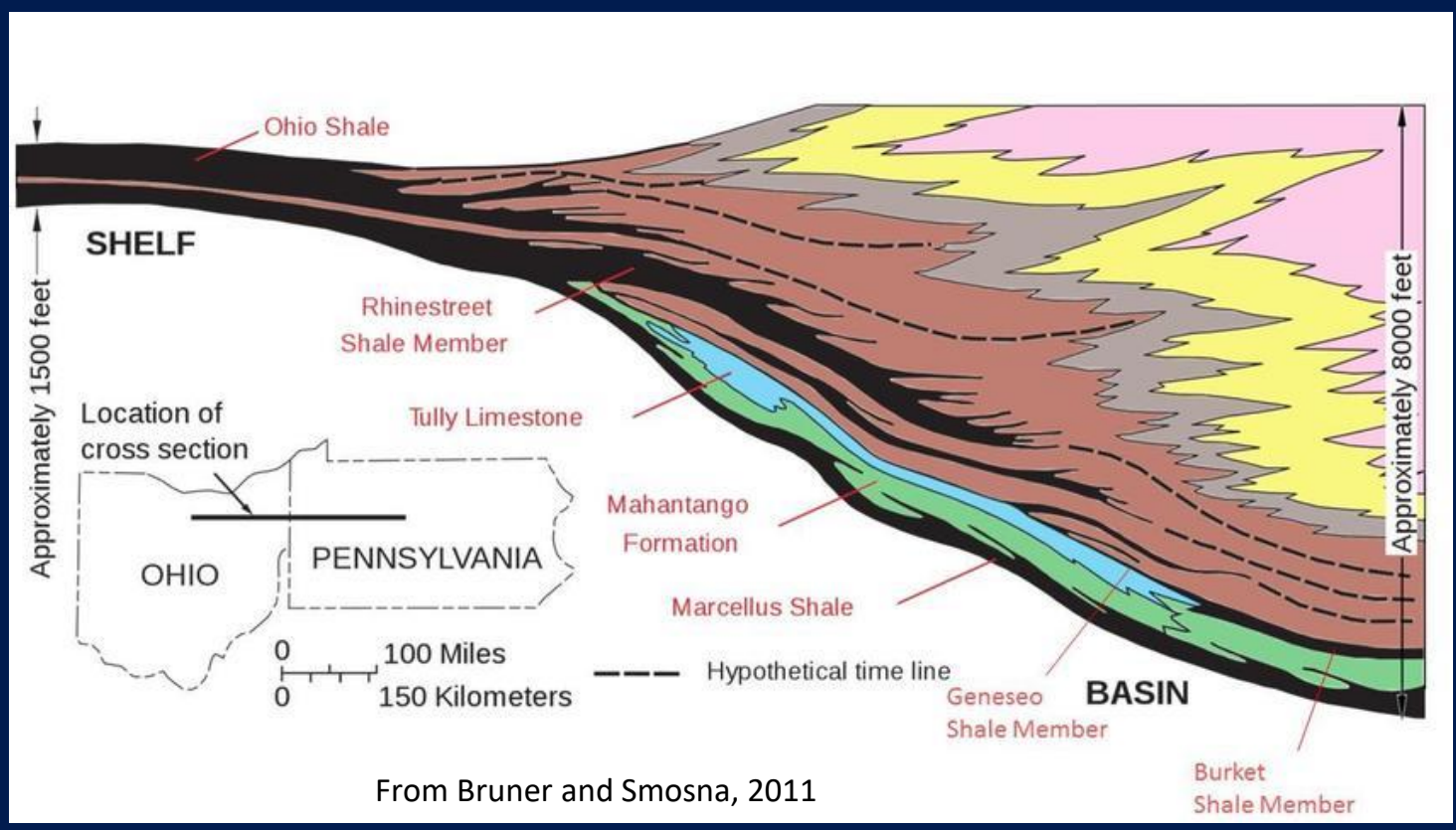

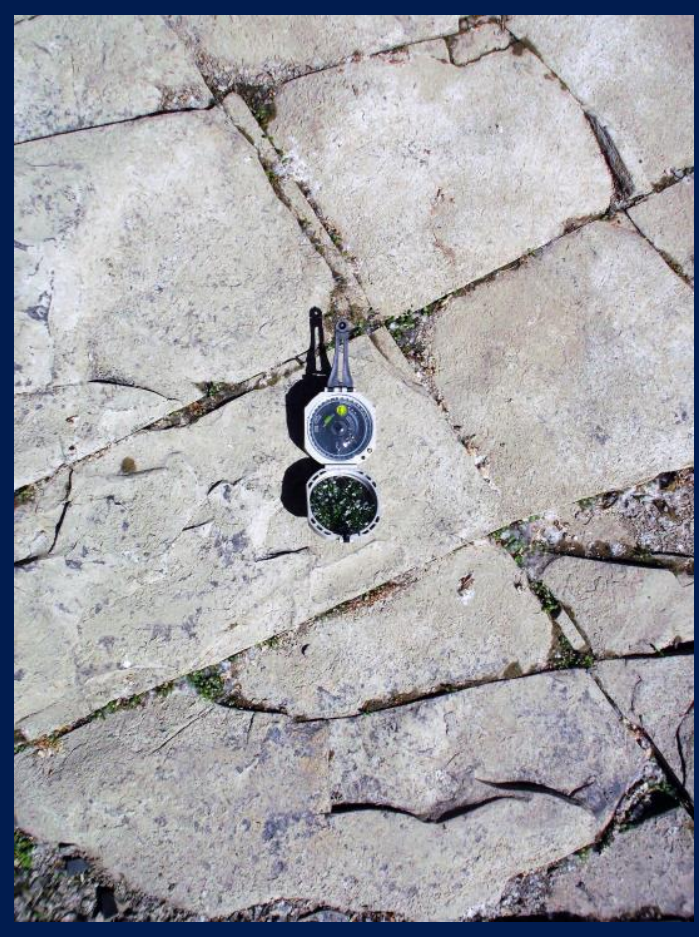

Photos by Dan Soeder: Oatka Creek (U) Seneca Quarry (L)

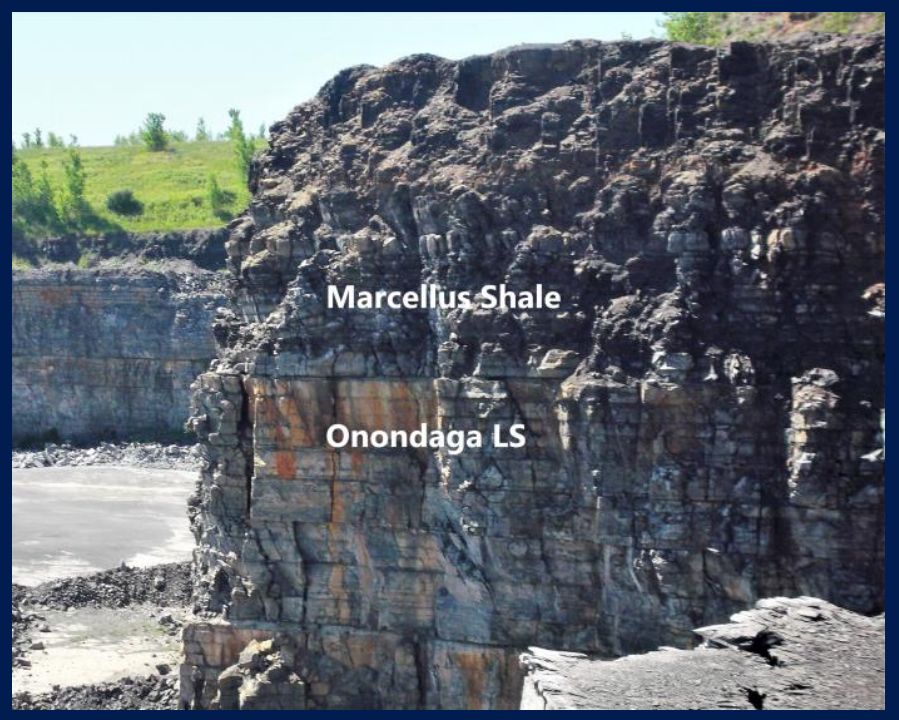




\section{//5. Bakken Formation}

Williston Basin

- Late Devonian to Early Mississippian black shale sandwich on limestone and sandstone.

- Conventional production since 1953.

- Completely subsurface; type section is in the H.O. Bakken No. 1 well, Williams Co., ND.

- Bakken and underlying Three Forks primarily produce light oil and associated gas.

- Developed in 2006 by EOG at Parshall.
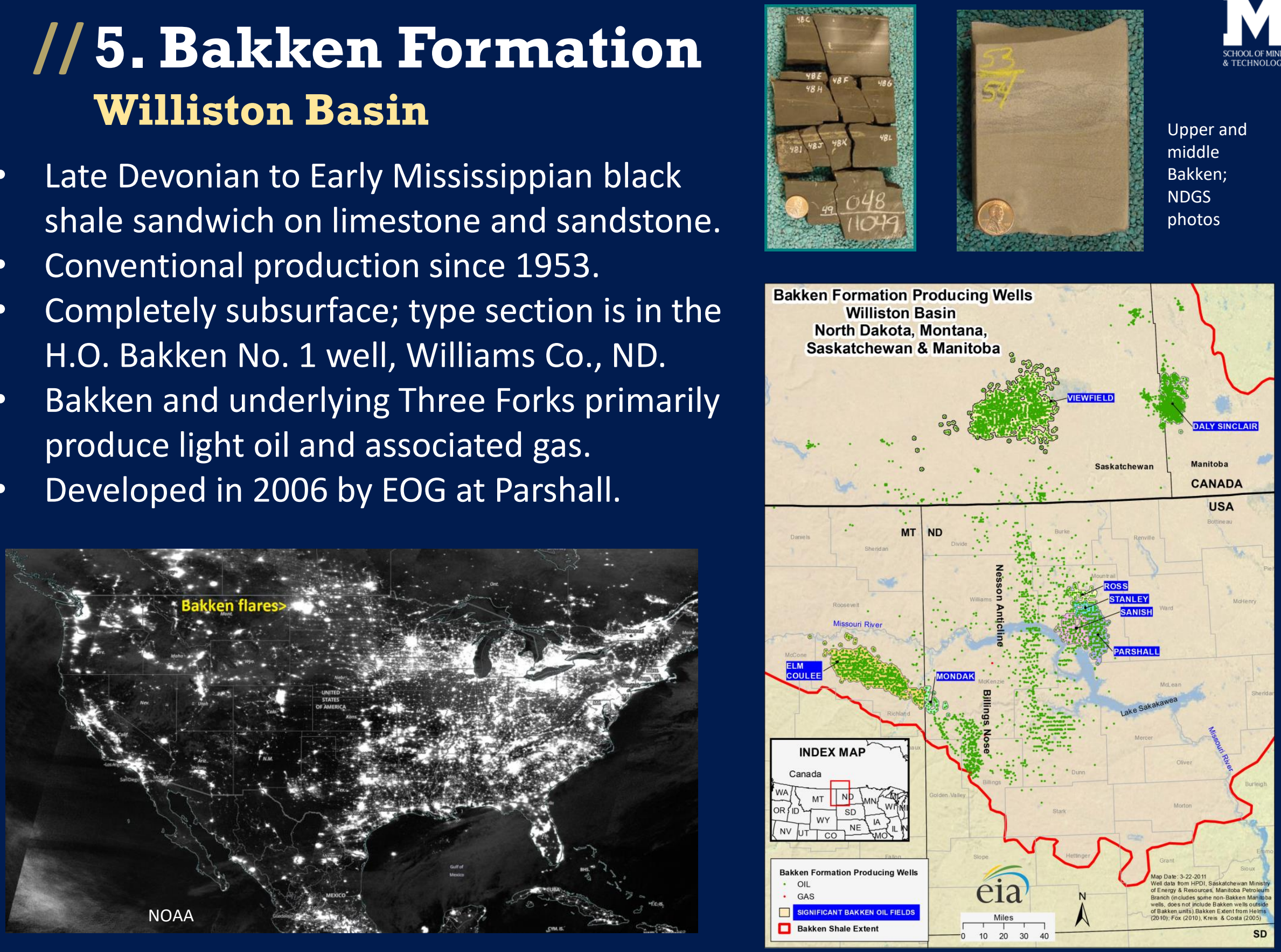


\section{// 6. Woodford Shale}

\section{Anadarko Basin}

- Late Devonian to Early Mississippian black, bituminous, cherty, fissile shale.

- Depth varies across basin; shale produces NGL shallow/dry gas deep.

- SCOOP/STACK are main plays.

- Newfield was primary developer.

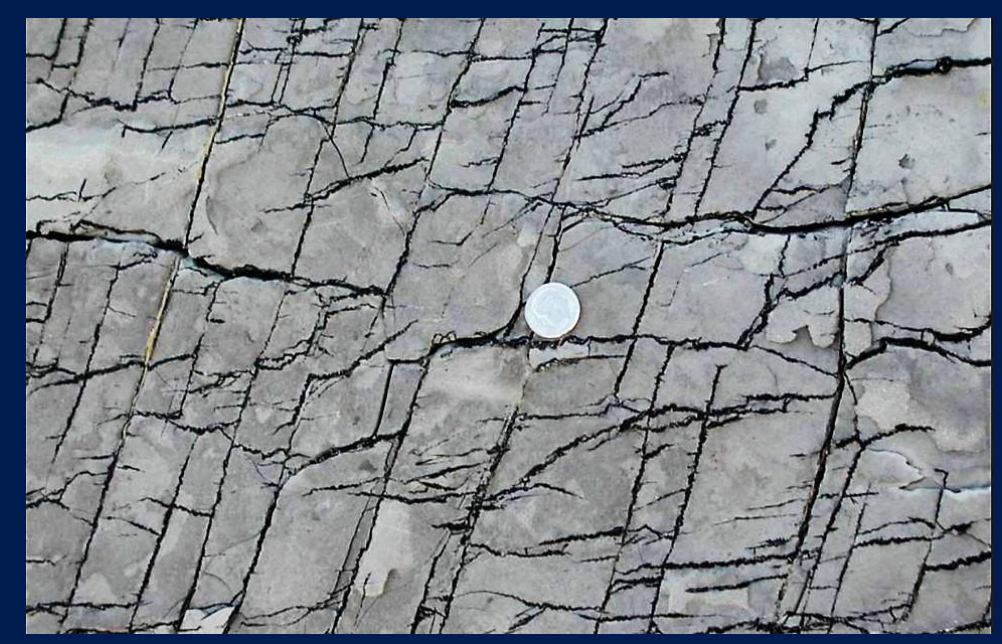

Bitumen-filled fractures in Woodford (OK Minerals)

South Central Oklahoma Oil Province

Sooner Trend Anadarko: Canadian and Kingfisher counties

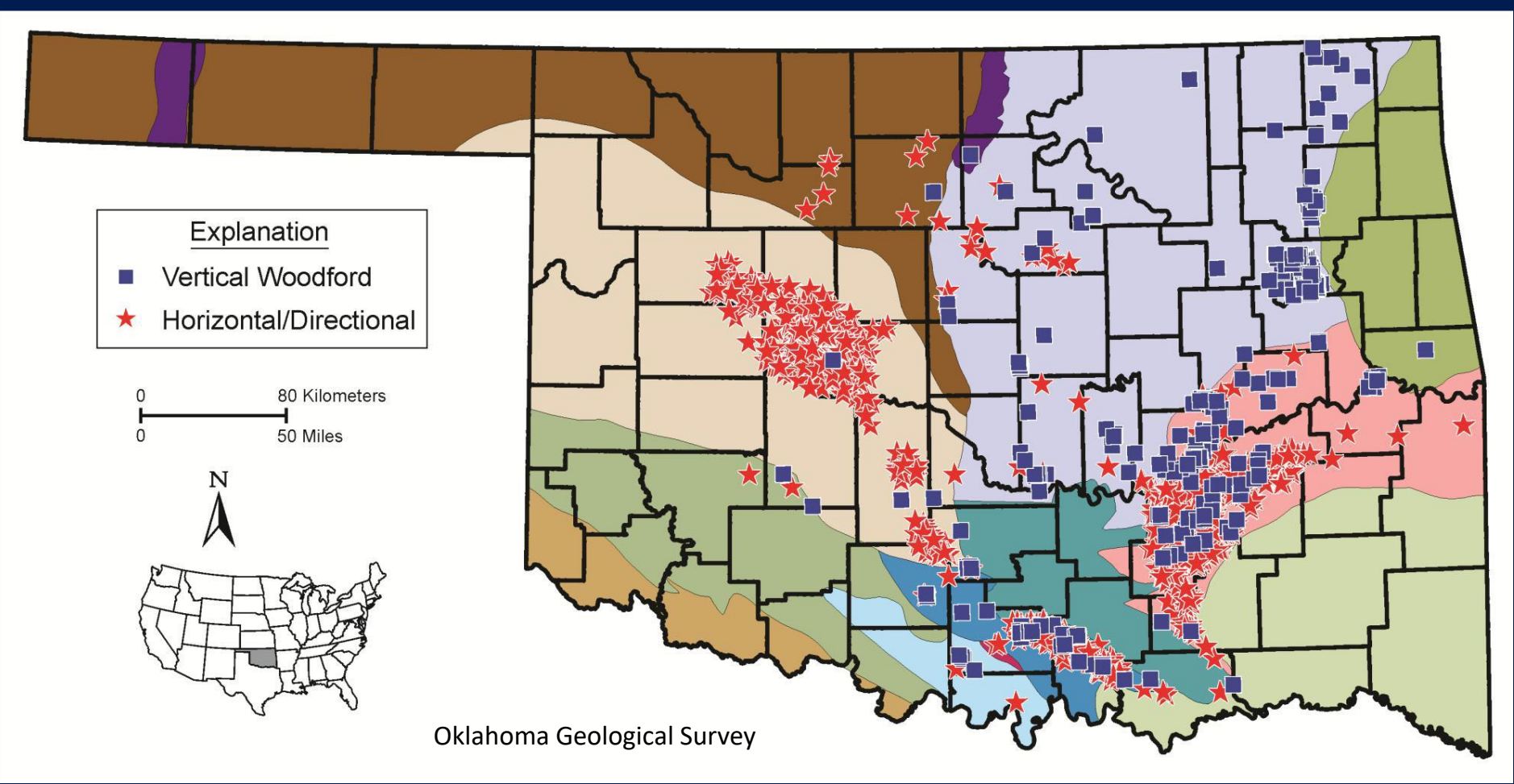




\section{// 7. Niobrara Formation}

\section{Denver-Julesburg Basin}

- Late Cretaceous chalk and calcareous shale in the Western Interior Seaway.

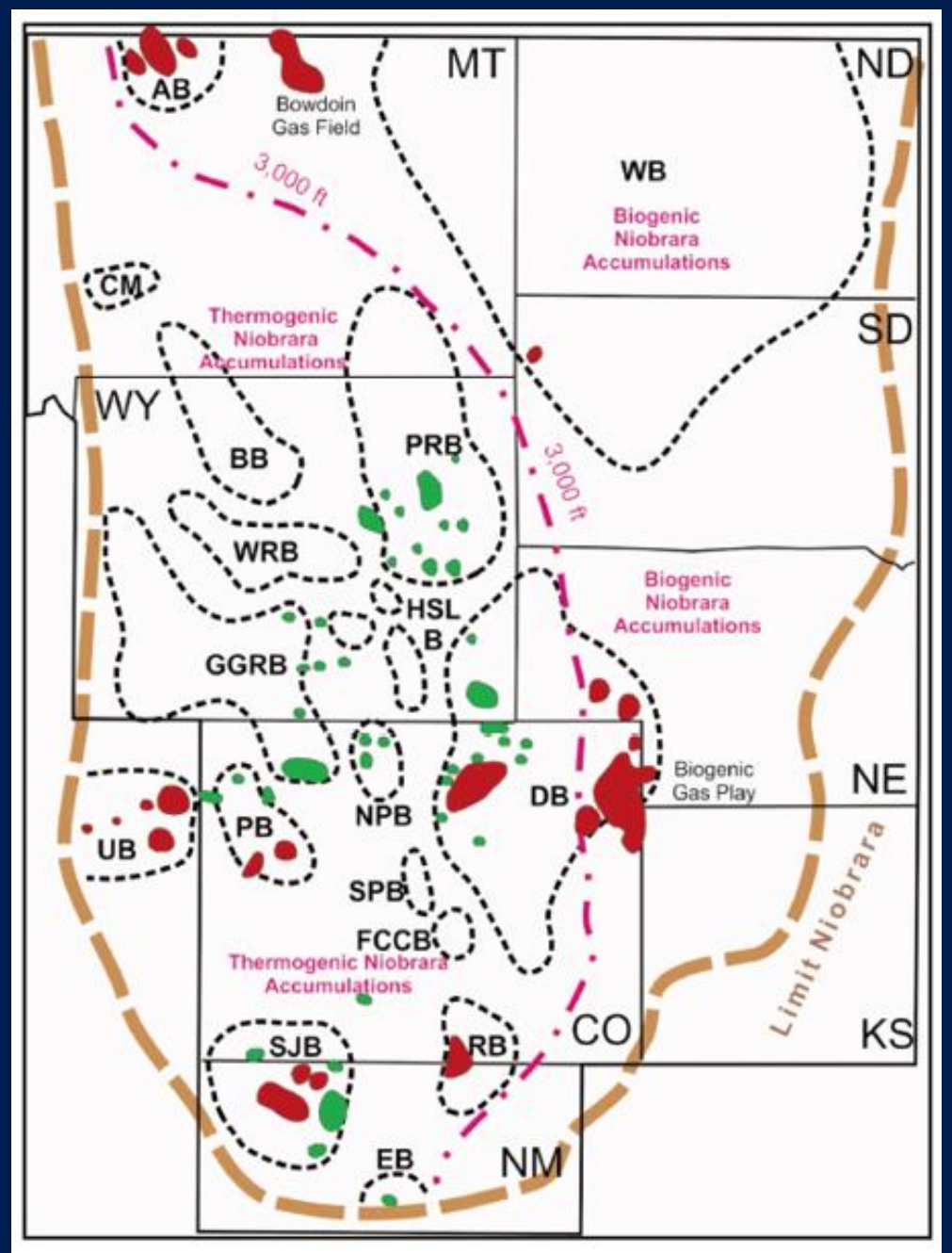

- Overlain by the organic-rich Pierre Shale.

- Mostly shallow, but thermally mature in deep structural basins.

- Developed by Whiting in eastern Colo.

- Makes part of stacked play in the PRB.

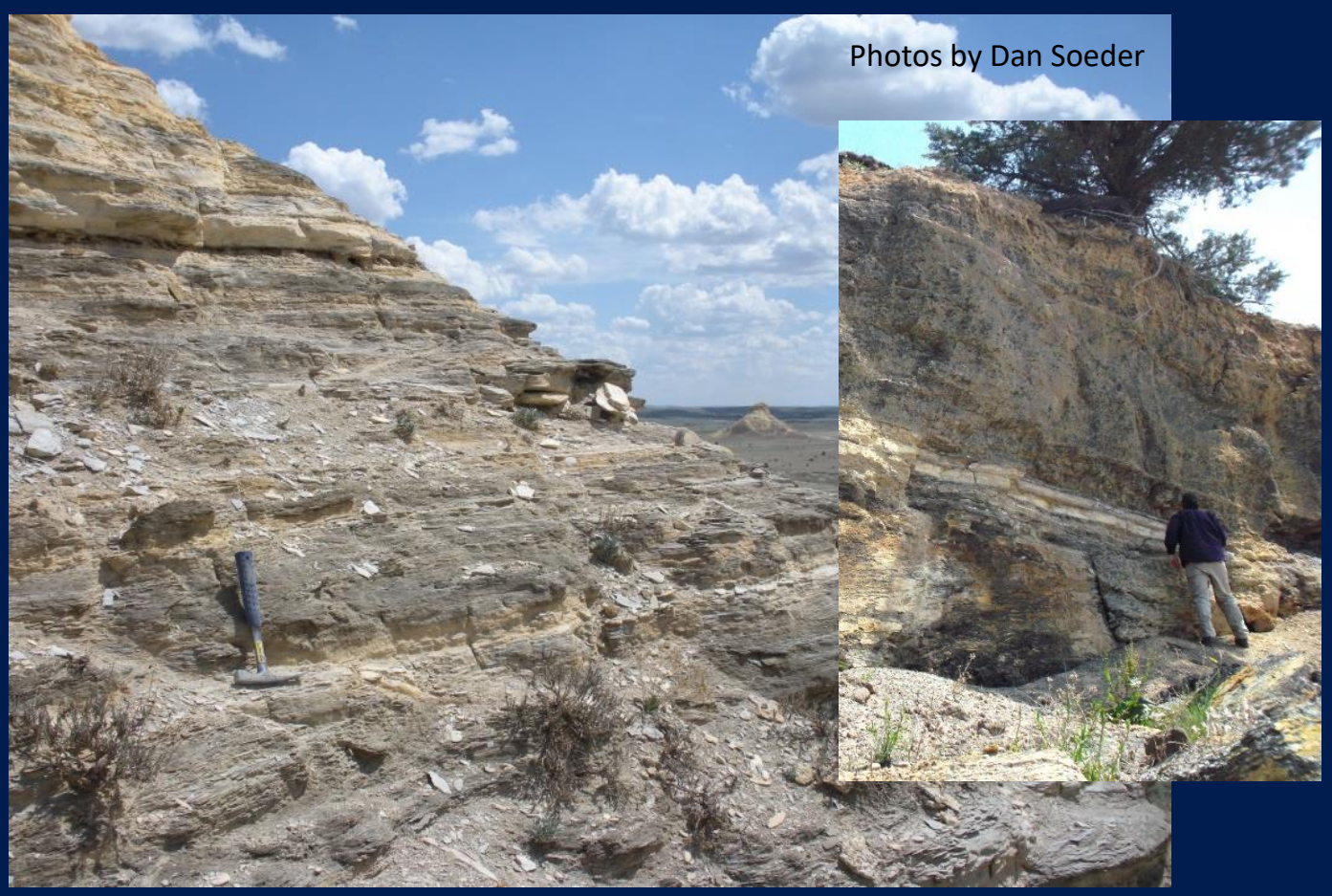




\section{// 8. Eagle Ford Shale}

\section{Texas Gulf Coast}

- Late Cretaceous calcareous shale on Gulf Coast from East Texas into Mexico.

- Located below the Austin Chalk and above the Buda Limestone and Woodbine.

- Varies in depth from outcrops to 14,000 feet toward the Gulf of Mexico.

- Produces oil and NGL shallow/dry gas deep; Petrohawk was primary developer.
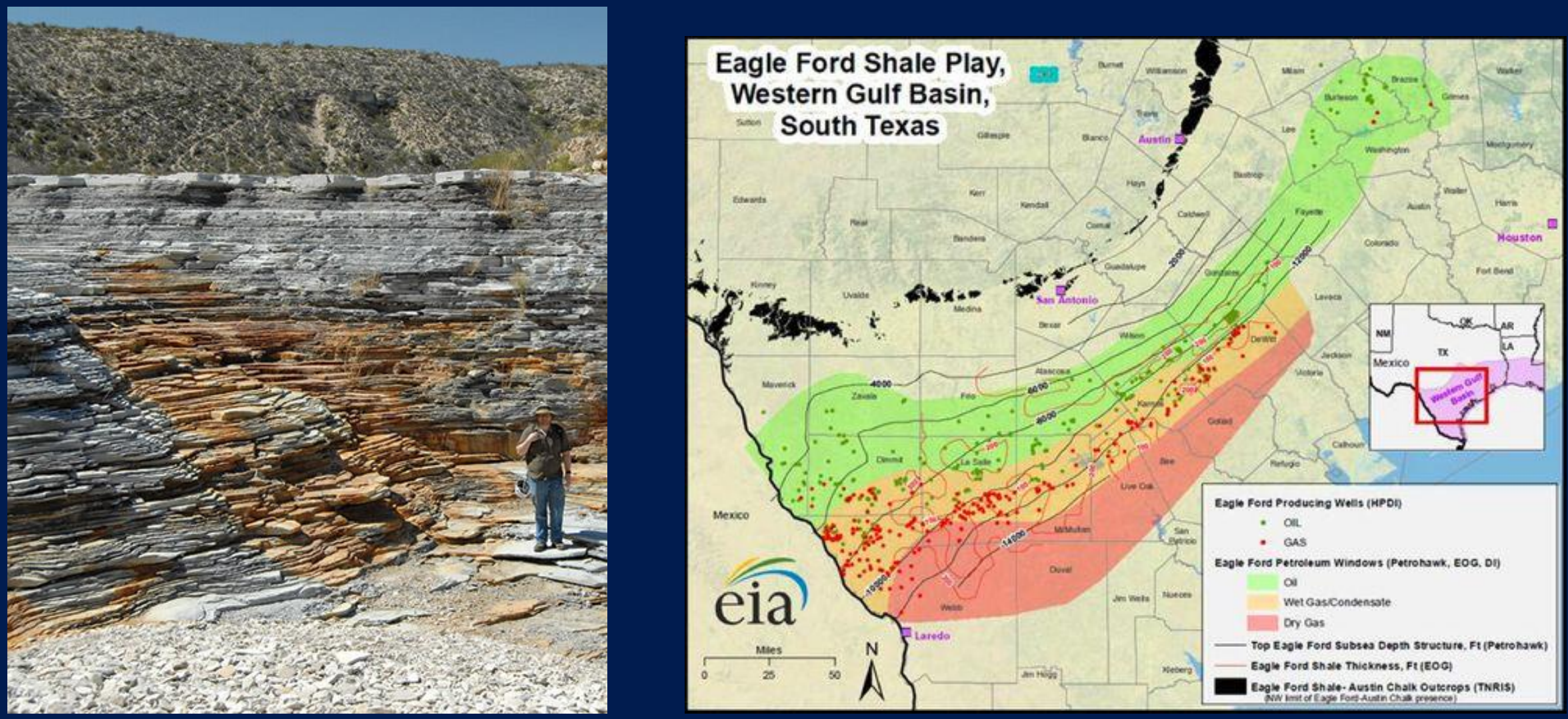


\section{// 9. Stacked Play}

Permian Basin

- Permian basin is located in the western part of Texas and southeastern New Mexico

- Two adjoining basins, the Delaware and the Midland, are separated by a platform.

- Conventional production since 1920.

- In 2010, unconventional formations created a large, stacked play.

1. Early Permian Wolfcamp Shale

2. Spraberry Sandstone ("Wolfberry")

3. Bone Spring Limestone ("Wolfbone")

4. Glorieta Sandstone

5. Yeso Formation

6. Middle Permian Delaware Mountain Group

- Currently running short of pipeline capacity.

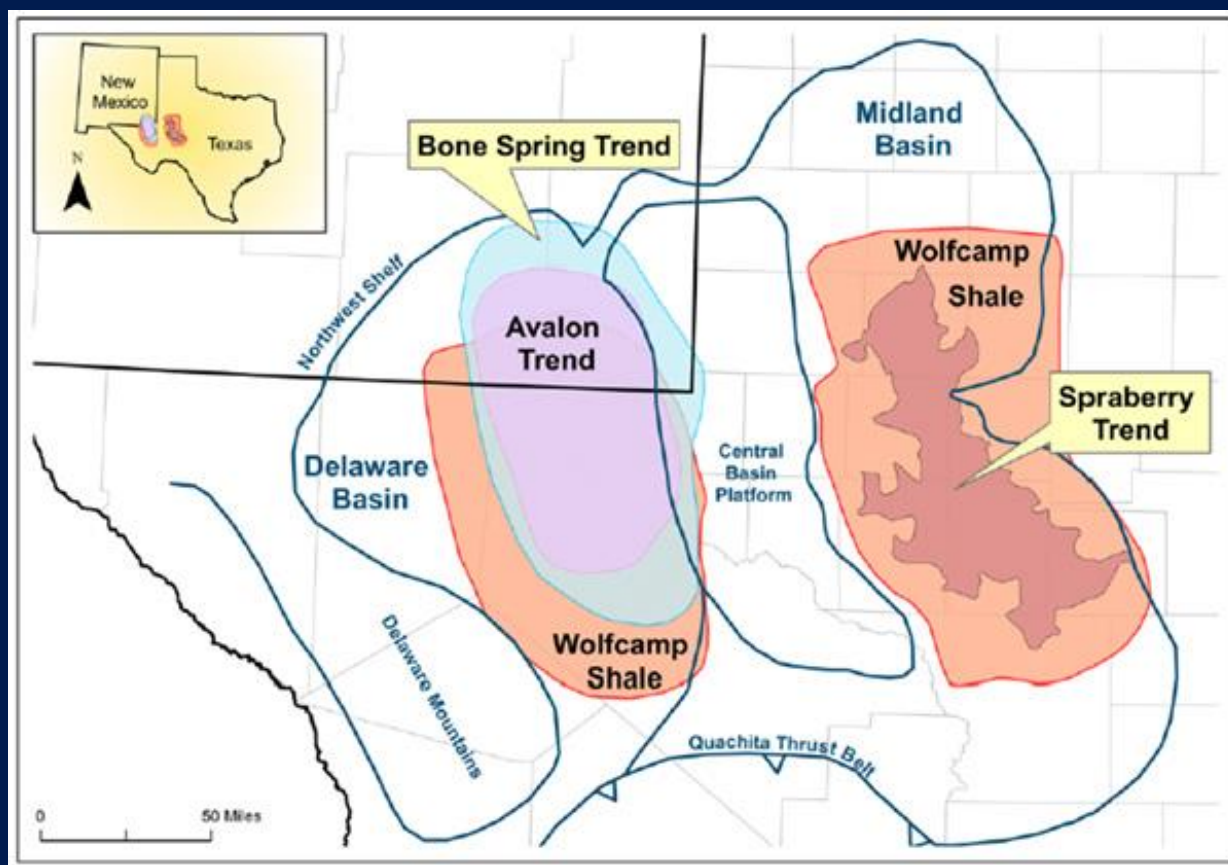

Permian Basin tight oil plays.

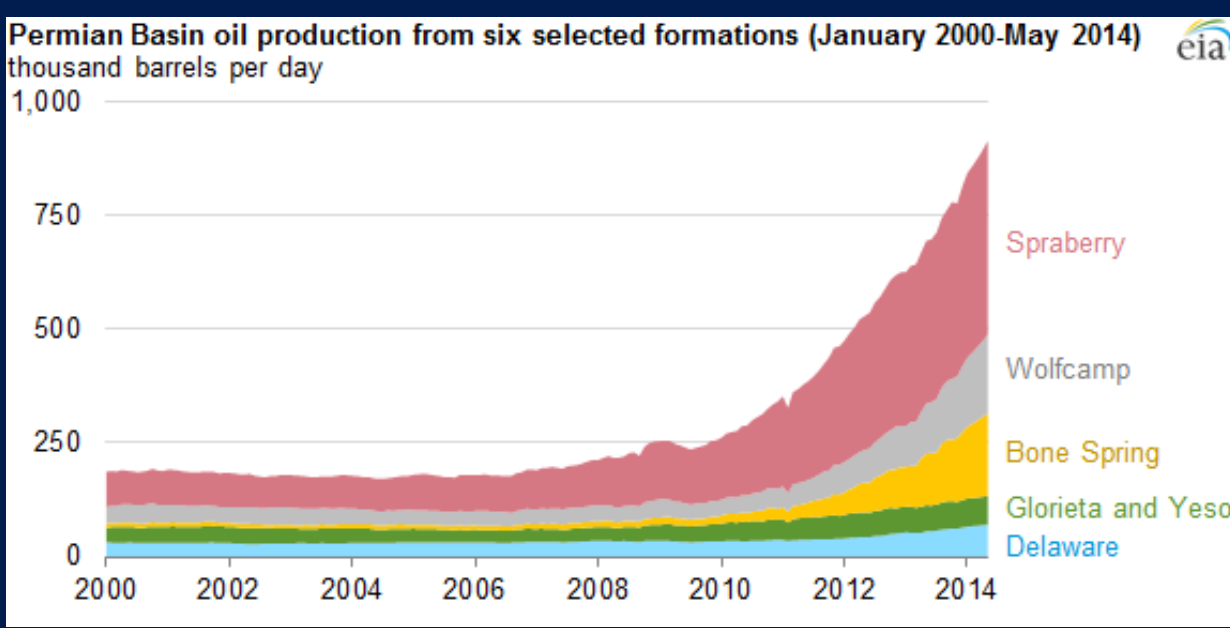




\section{// 10. Utica-Point Pleasant Shale}

\section{Appalachian Basin}

- Middle Ordovician Utica Shale overlies Trenton-Black River limestones throughout the Appalachian basin.

- Production began in 2011-2012 from dual-completion wells with Marcellus in western PA. Chesapeake is by far the major developer.

- Focus shifted to southeastern Ohio where Utica is rich in NGL.

- Record-setting wells (19,500 ft lateral, $125+$ stages, IP=73 MMcf/d)

- Point Pleasant is equivalent formation that outcrops in KY; Ohio GS uses both.
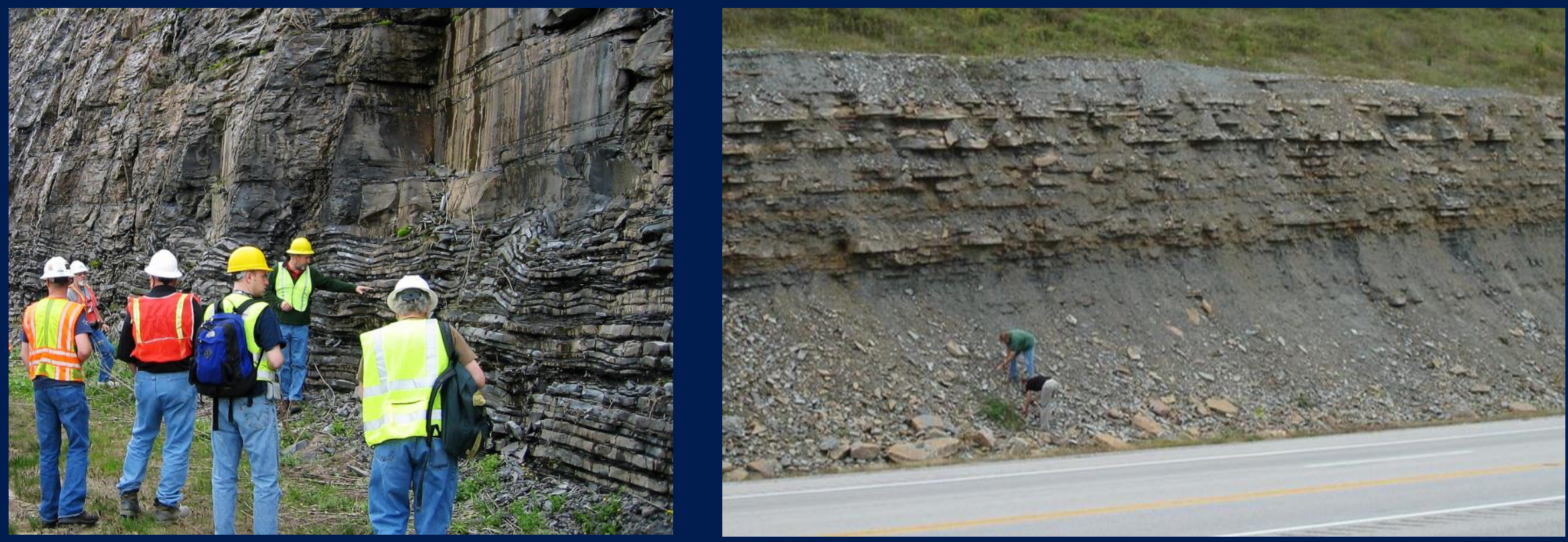


\section{// Shale Gas Development Emerging U.S. Plays}

\begin{tabular}{|c|c|c|c|c|c|c|}
\hline & & & & & & \\
\hline Formations & Age & $\begin{array}{l}\text { Basins \& } \\
\text { Location }\end{array}$ & Rock Type & Production & $\begin{array}{l}\text { Potential } \\
\text { Resource }\end{array}$ & Problems \\
\hline Granite Wash & Pennsylvanian & Anadarko, OK & Submarine fans & Oil, NGL, gas & 114 billion $\mathrm{BOE}$ & $\begin{array}{l}\text { Very complex } \\
\text { geology }\end{array}$ \\
\hline Austin Chalk & Late Cretaceous & Texas Gulf Coast & Chalk \& marl & Oil, NGL, gas & $\begin{array}{l}4.1 \text { billion bbl oil } \\
18 \text { TCF natural gas } \\
1 \text { billion bbl NGL }\end{array}$ & $\begin{array}{l}\text { Brittle, hard to } \\
\text { drill and frack }\end{array}$ \\
\hline Tuscaloosa Trend & Late Cretaceous & $\begin{array}{l}\text { Louisiana- } \\
\text { Mississippi border } \\
\text { region }\end{array}$ & Marine shale & Oil, NGL & $\begin{array}{c}\text { "comparable to the } \\
\text { Eagle Ford" }\end{array}$ & $\begin{array}{c}\text { Too soft to frack } \\
\text { well, crumbles }\end{array}$ \\
\hline Upper Devonian & Late Devonian & $\begin{array}{c}\text { Appalachian, WV, } \\
\text { PA }\end{array}$ & Black shale & Dry gas & 11 TCF gas & $\begin{array}{l}\text { Shallow, less } \\
\text { productive }\end{array}$ \\
\hline Rogersville Shale & Middle Cambrian & $\begin{array}{c}\text { Rome Trough, } \\
\text { Appalachian, KY- } \\
\text { WV }\end{array}$ & $\begin{array}{l}\text { argillaceous shale } \\
\text { \& limestone }\end{array}$ & Dry gas & $\begin{array}{c}\text { "comparable to the } \\
\text { Marcellus" }\end{array}$ & $\begin{array}{c}\text { Very deep and } \\
\text { expensive to } \\
\text { drill }\end{array}$ \\
\hline Atlantic Rift Basins & Mesozoic & $\begin{array}{c}\text { Atlantic Coastal } \\
\text { Plain }\end{array}$ & Rift basin fill & Gas, NGL & $\begin{array}{c}3.86 \text { TCF gas; } 135 \\
\text { million bbl NGL }\end{array}$ & $\begin{array}{l}\text { Strong local } \\
\text { opposition }\end{array}$ \\
\hline Monterey Formation & $\begin{array}{l}\text { Middle to Late } \\
\text { Miocene }\end{array}$ & $\begin{array}{l}\text { San Joaquin basin, } \\
\text { California }\end{array}$ & $\begin{array}{l}\text { organic-rich, } \\
\text { siliceous shale }\end{array}$ & Oil, NGL, gas & $\begin{array}{c}21 \text { million bbl oil } \\
27 \text { BCF gas } \\
1 \text { million bbl NGL }\end{array}$ & $\begin{array}{c}80 \text { dry holes, } \\
\text { shale may be } \\
\text { spent }\end{array}$ \\
\hline $\begin{array}{l}\text { Shublik Formation, } \\
\text { Kingak Shale, and } \\
\text { Brookian shale }\end{array}$ & $\begin{array}{l}\text { Triassic to } \\
\text { Cretaceous }\end{array}$ & $\begin{array}{l}\text { North Slope, } \\
\text { Alaska }\end{array}$ & $\begin{array}{l}\text { limestone, shale, } \\
\text { siltstone, and tuff }\end{array}$ & Oil, NGL, gas & $\begin{array}{c}2 \text { billion bbl oil } \\
500 \text { mill bbl NGL } \\
80 \text { TCF gas }\end{array}$ & $\begin{array}{l}\text { Economics of } \\
\text { Arctic location }\end{array}$ \\
\hline
\end{tabular}




\section{// Summary and Conclusions}

Shale gas production as a

percent of total gas production

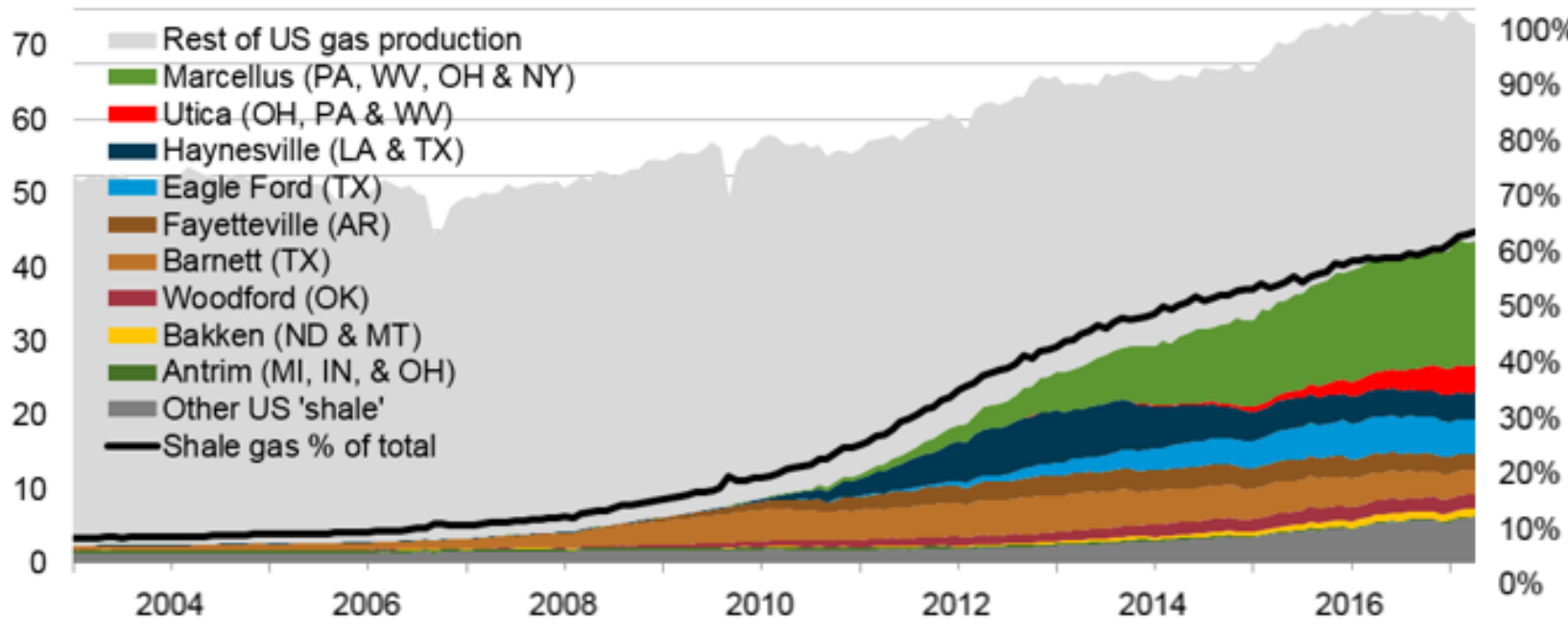

Tight oil production

Tight oil production as a million barrels of oil per day percent of total oil production

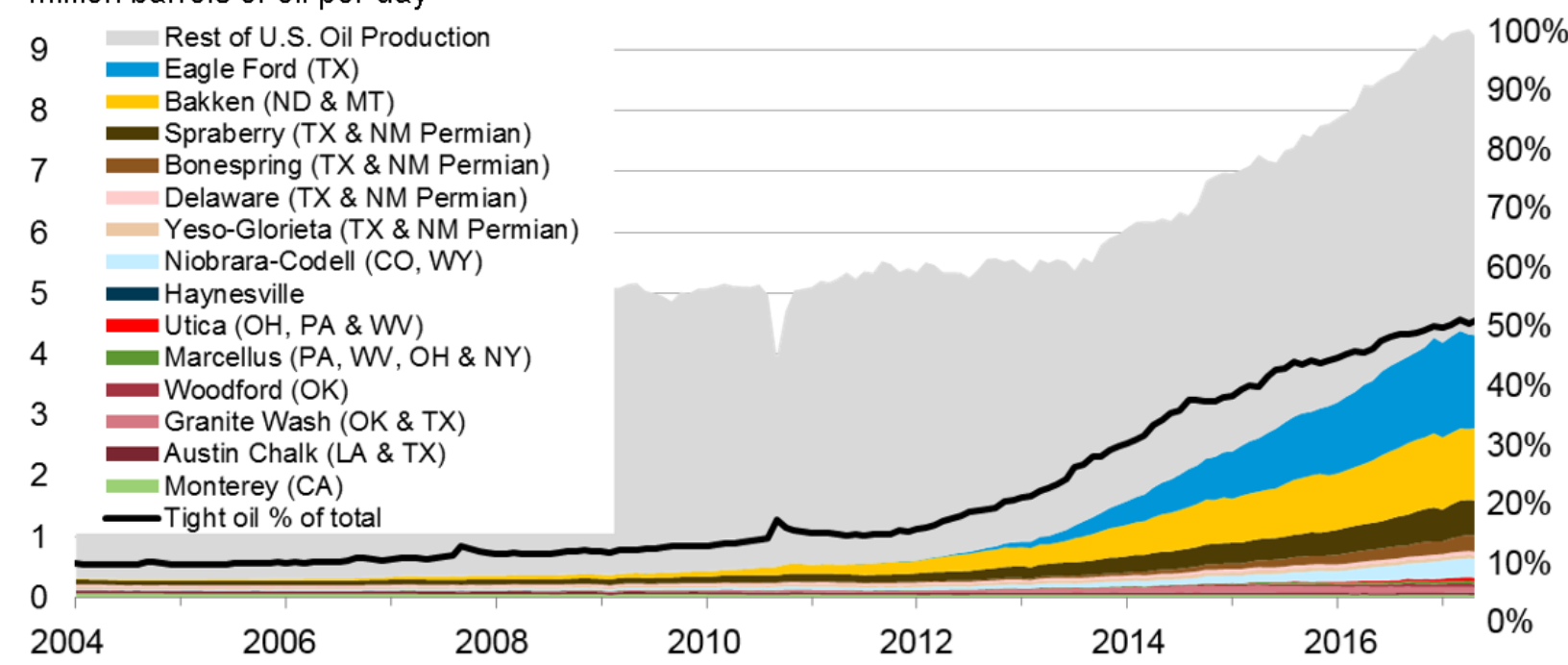

- $\quad$ The single largest gas producing formation in the United States is the Marcellus Shale.

- The second-largest oil producing state in the U.S. is North Dakota due to the Bakken Shale.

- The largest oil producing state in the U.S. is Texas due to tight oil from the Eagle Ford and Permian Basin.

- Source: U.S. Energy Information Administration (Popova et al, 2018) 